\section{Komplexe Krankheitsbilder}

Mit der neuen Auflage nach fast zehn Jahren ist das bekannteste Syndrom-Buch wieder aktualisiert und entscheidend verbessert worden. Der Inhalt ist mit einem neuen Farbleitsystem noch übersichtlicher aufgestellt und umfasst als Hauptkapitel u.a. folgende Inhalte, die bereits eine erste Orientierung ermöglichen: morphologische Auffälligkeiten, exogene Ursachen, chromosomale Störungen, Syndrome mit Kontrakturen, Kraniostenose-Syndrome, Hochwuchs- und Adipositas-Syndrome, proportionierte Kleinwuchs-Syndrome, Syndrome mit isolierter ZNS-Fehlbildung, Syndrome mit fazialen und Extremitätenfehlbildungen, Osteochondrodysplasien etc.

Das Wissen über die kaum übersehbare Vielzahl von Syndromen ist in der täglichen Praxis manchmal recht schmerzlich begrenzt. Hier kann dieses Buch mit seiner gut gegliederten Sammlung eine große Hilfe sein, ob man nun den aktuellen Patienten vergleicht oder Details zu einem schon bekannteren Krankheitsbild sucht. Das Buch zeigt auch kleinere morphologische Auffälligkeiten auf und liefert so die Bausteine für eine Beschreibung komplexerer Krankheitsbilder.

Im Hauptteil findet man wichtige Merkmale von fast 400 aufgefächerten Syndromen. Änderungen im Laufe des Lebens sind in Fotos aus den verschiedenen Altersabschnitten berücksichtigt. Das Buch hilft beim genauen Hinschauen und der akribischen Erfassung und fördert so das kritische, abschließende Urteil. So ist ein wichtiger Atlas für Ärzte vieler Fachrichtungen zu einem erschwinglichen Preis jetzt wieder auf dem Markt, und wird v.a. für Pädiater, Humangenetiker und Gynäkologen von großem Nutzen sein, zum Wohle der betroffenen Kinder und ihrer Familien.

\section{Dr. Friedrich Grüttner}

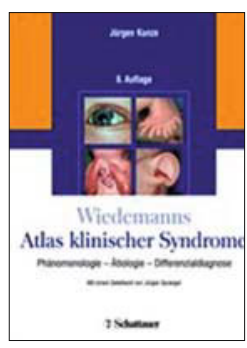

Jürgen Kunze

Wiedemanns Atlas klinischer Syndrome Schattauer Verlag, Stuttgart 2010 $269,00 €$ ISBN: 978-3-79452657-4

\section{Unternehmen Arztpraxis}

Wenn das „Unternehmen Arztpraxis“ florieren soll, bilden bekanntermaßen die Aspekte Betriebswirtschaft, Kooperationsformen, Steuer-/Vertragsarzt-/Arbeits-/ Haftungsrecht, Marketing, Mitarbeiterführung, Praxisorganisation und auch die Altersvorsorge die Basis. Meist wird aber übersehen, dass erst das sinnvolle Zusammenspiel und die Wechselwirkungen aller dieser Aspekte ein langfristiges Gesamtkonzept bilden.

Dies führen die Autoren - alle drei erfahrene Experten auf ihrem Gebiet - klar verständlich vor Augen: nicht nur für Einsteiger, die eine Praxis gründen oder übernehmen wollen, sondern auch für Niedergelassene, die ihr Praxiskonzept optimieren und ihr „Management-Wissen“ entsprechend den aktuellen und zukünftigen (Konkurrenz-) Situationen auffrischen und erweitern wollen.

red

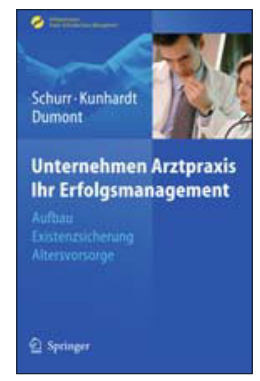

M. Schurr et al.

Unternehmen Arztpraxis - Ihr Erfolgsmanagement Springer, Heidelberg 2009 $39,95 €$ ISBN: 978-3-54048559-9

\section{Navigation durch die Neuropädiatrie}

Getreu der Devise „Make things as simple as possible, but not simpler" von Albert Einstein kommen die Autoren in diesem Buch dem chronischen Zeitmangel der Ärzte entgegen: Mit Hilfe der sogenannten Paediatric Clinical Scouts (PCS) werden kondensiert in Stichworten Informationen über die einzelnen Krankheitsbilder der Neuropädiatrie vermittelt. Die Scouts dienen somit dem Leser als eine Art Navigationskarten. Horizontal von links nach rechts wird er von der Anamnese über die Diagnostik bis zur Diagnose geführt. In der vertikalen Ebene erfährt er Inhalte der Anamnese und Diagnostik. Der PCS-Diagnostik folgt die
PCS-Therapie. Für die Inhalte der PCS sowie des vollständigen Werks gilt: so knapp wie möglich, Wichtiges vor Unwichtigem, Häufiges vor Seltenem. Websites, Leitlinien, Hinweise auf Selbsthilfegruppen und aktuelle Cochrane-Rewiews erlauben eine interdisziplinäre, evidenzbasierte Wertung.

Die Knappheit des Texts mag von einigen geschätzt werden. Um ein Krankheitsbild besser kennenzulernen, wird der Arzt aber nicht umhinkönnen, auf konventionelle Lehrbücher zurückzugreifen. Denn, um das Buch zu nutzen, benötigt der Leser bereits ein solides neuropädiatrisches Wissen. Lediglich in den Kapiteln Kopfschmerz, Schwindel und Skoliose wird er vom Symptom zur Diagnose geführt. In den anderen Kapiteln kann er die von ihm gestellte Diagnose anhand der aufgeführten Kriterien lediglich überprüfen. Gewöhnlich kommt der Patient jedoch nicht mit der Diagnose in die Praxis, sondern mit Symptomen, die mit Hilfe von Anamnese, klinischer Untersuchung und dem Wissen des Arztes zur Diagnose führen. Auch bleibt bei der Auflistung der Symptome einzelner Krankheitsbilder offen, wie häufig diese auftreten. Beispielsweise erfährt der Leser, dass es im Verlauf des RETT-Syndroms zu epileptischen Erscheinungen und im Jugendalter noch einmal zu einer deutlichen Abnahme der Gehfähigkeit kommt. An dieser Stelle wären Angaben zur Häufigkeit wichtig, da die Eltern des Kindes wissen werden wollen, wie wahrscheinlich eine Epilepsie ist und ob alle Kinder im Rollstuhl enden - etwas mehr Text wäre also wünschenswert.

Das Buch mag sich insgesamt als Nachschlagewerk für den Neuropädiater in Klinik und Praxis eignen. Ob es einen zusätzlichen Vorteil zu den bereits auf dem Markt befindlichen Neuropädiatrie-Büchern darstellt, halte ich für fraglich.

Dr. Kirsten Stollhoff

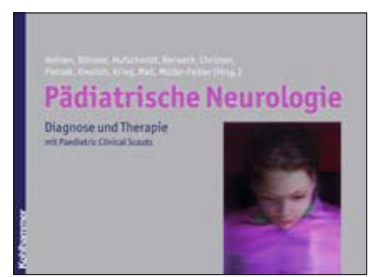

Florian Heinen et al.

\section{Pädiatrische Neurologie}

Kohlhammer Verlag, Stuttgart 2009

$199,00 €$

ISBN 978-3-17-019468-7 